\title{
Neonatal Dexamethasone Treatment in the Rat Leads to Kidney Damage in Adulthood
}

\author{
WILLEM B. DE VRIES, PLEUNIE VAN DEN BORNE, ROEL GOLDSCHMEDING, ROEL A. DE WEGER, MIRIAM P. BAL, \\ FRANK VAN BEL, AND MATTHIJS F.M. VAN OOSTERHOUT \\ Departments of Neonatology [W.B.V., M.P.B., F.B.] and Pathology [P.B., R.G., R.A.W., M.F.M.O.], University Medical Center \\ Utrecht/Wilhelmina Children's Hospital, Utrecht, 3508 AB, The Netherlands
}

\begin{abstract}
Recently, concern has been raised that corticosteroid treatment of preterm neonates might be associated with adverse effects later in life, including early development of hypertension. Here, we investigate the impact of neonatal dexamethasone (Dex) treatment on early renal cell proliferation and nephron number. We analyzed mitotic activity in renal cortex of rat pups neonatally treated with Dex. Nephron number was measured and possible renal damage was quantified by counting inflammatory foci, ED-1 positive cells (macrophages), and the desmin score (activated podocytes). Mitotic activity was 34 and 29\% lower on d 2 and 4 in Dex-treated rats compared with saline-treated controls. The number of glomeruli was lower at $4 \mathrm{wk}$, but nephron size was unchanged after Dex treatment, as calculated from glomerular density and (lower) body- and kidney weight. At wk 50, the glomerular number was significantly lower in Dex-treated rats, whereas body and kidney weight were the same as in Sal controls. Dex rats also showed more kidney damage, manifested by a $\sim 3.5$-fold increase in inflammation foci/ $\mathrm{mm}^{2}$ and in ED-1 positive cells $/ \mathrm{mm}^{2}$ and a $\sim 4.3$-fold increased desmin score. Temporary suppression of mitotic activity during neonatal Dex treatment leads to reduction of nephron number and more kidney damage later in life. (Pediatr Res 67: 72-76, 2010)
\end{abstract}

$\mathrm{P}$ reterm infants suffering from severe respiratory distress syndrome have a high risk of developing chronic lung disease (CLD) due to a proinflammatory reaction occurring in immature lungs $(1,2)$. Glucocorticoids, predominantly dexamethasone (Dex), are widely used to prevent or reduce this complication because of their anti-inflammatory properties $(3,4)$. However, recent animal and human studies showed that neonatal Dex treatment is accompanied with several adverse effects, short term as well as long term, and concern arose with respect to the development of important organ systems and neurodevelopmental outcome $(3,5-11)$. Furthermore, neonatal Dex treatment in rats was associated with a poor survival (12).

Recently, our group reported on the effects of neonatal Dex treatment on the development of the heart. It was shown that in rats a 3-d course of Dex significantly suppressed proliferative activity of cardiomyocytes in the first week of life. At 50

Received February 12, 2009; accepted August 26, 2009

Correspondence: Willem B. de Vries, M.D., Ph.D., Department of Neonatology, Room KE.01.123.1, Wilhelmina Children's Hospital, PO Box 85090, Utrecht, 3508 AB, The Netherlands; e-mail: w.b.devries-3@umcutrecht.nl

Supported by grants NHS 2001B081 (to M.P.B.) and NHS 2004 T31 (to M.F.M.O.) from the Netherlands Heart Foundation.

W.B.V. and P.B. contributed equally to the article.

M.F.M.O. is currently at Department of Pathology, St. Antonius Hospital Nieuwegein, 3430 EM, The Netherlands. wk of age this resulted in a lower number of cardiomyocytes in the heart, cardiomyocyte hypertrophy and increased interstitial fibrosis (13-15).

However, not much is known about the effects of Dex on the kidney. In a survival study, Kamphuis et al.(12) found that neonatally Dex-treated rats showed more signs of kidney damage, like glomerulosclerosis and cysts, in global histopathological examination. However, in this study, no quantitative measurements of renal damage were done.

Most other studies concerning effects of glucocorticoids on the kidney focused only on the prenatal treatment of Dex. One study (16) indicated that neonatal birth weight was significantly lower in both male and female rats after prenatal Dex treatment, whereas kidney weight at $70 \mathrm{~d}$ was significantly lower in their Dex-treated rats. The nephron number in the Dex-treated rats was significantly lower compared with the untreated control rats (16). Ortiz et al.(17) found that maternal Dex treatment at d 15 and 16 of rat pregnancy leads to a $30 \%$ reduction of nephrons in the offspring compared with the untreated controls. These studies indicate that prenatal Dex may have potentially negative effects like a reduction in nephron number. However, to the best of our knowledge, studies concerning the effects on the kidney of neonatal Dex are scarce (18).

Therefore, the aim of this study was to investigate shortand long-term effects of neonatal Dex treatment on the development of the kidney and to look for possible Dex-induced renal damage. First, we aimed to investigate the proliferative activity in renal cells in rat pups, in analogy with our earlier studies that observed suppression of proliferative activity in the heart (14). Second, we investigated whether changes in proliferative activity may be associated with changes in glomerular density later in life. Finally, we investigated whether Dex treatment results in kidney damage at middle age (50wk-old rats).

\section{MATERIALS AND METHODS}

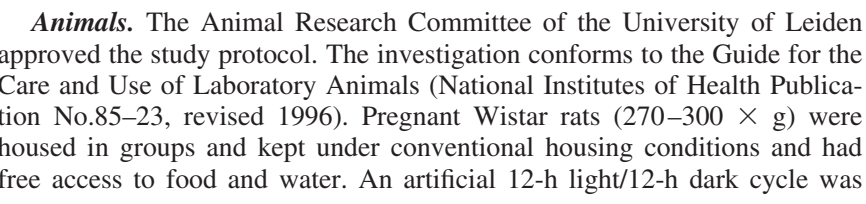

Abbreviations: CLD, chronic lung disease; Dex, dexamethasone; Sal, saline 
Table 1. Anatomical parameters of 2-, 4-, and 7-d-old rats

\begin{tabular}{|c|c|c|c|c|c|c|}
\hline \multirow{2}{*}{$\begin{array}{c}\text { Age } \\
\text { Treatment }(n)\end{array}$} & \multicolumn{2}{|r|}{ Day 2} & \multicolumn{2}{|r|}{ Day 4} & \multicolumn{2}{|r|}{ Day 7} \\
\hline & Sal (8) & Dex (8) & Sal (9) & Dex (8) & Sal (7) & Dex (6) \\
\hline Body weight $(\mathrm{g})^{*}$ & $7.60 \pm 0.37$ & $6.95 \pm 0.49(p=0.012)$ & $9.73 \pm 0.38$ & $6.43 \pm 0.71(p=0.002)$ & $13.78 \pm 1.08$ & $10.66 \pm 1.58(p=0.016)$ \\
\hline Kidney weight (mg) & $53.75 \pm 6.94$ & $52.50 \pm 3.78(\mathrm{~ns})$ & $65.56 \pm 6.82$ & $55.63 \pm 6.78(p=0.021)$ & $94.29 \pm 9.32$ & $80.00 \pm 10.49(p=0.022)$ \\
\hline
\end{tabular}

Data are presented as mean $\pm \mathrm{SD}(p$ Dex $v s$ Sal).

* Published by de Vries et al., (14).

ns, not significant.

Table 2. Anatomical parameters of 4-wk-old and 50-wk-old rats

\begin{tabular}{lcccccc}
\hline & \multicolumn{3}{c}{ Week 4} & & \multicolumn{2}{c}{ Week 50 } \\
\cline { 2 - 3 } \multicolumn{1}{c}{ Treatment $(\mathrm{n})$} & Sal $(8)$ & Dex $(8)$ & Sal $(7)$ & Dex $(6)$ \\
\hline Body weight $(\mathrm{g})$ & $85.87 \pm 8.48$ & $72.22 \pm 3.21(-16 \%, p=0.0006)$ & & $521.29 \pm 37.90$ & $522.67 \pm 31.44(\mathrm{~ns})$ \\
Kidney weight $(\mathrm{g})$ & $0.46 \pm 0.04$ & $0.35 \pm 0.03(-24 \%, p=0.0002)$ & & $1.68 \pm 0.25$ & $1.61 \pm 0.29(-4 \%, \mathrm{~ns})$ \\
Number of glomeruli/kidney & $177 \pm 16.37$ & $145.88 \pm 10.41(-18 \%, p=0.0003)$ & & $205.71 \pm 19.41$ & $165 \pm 23.19(-20 \%, p=0.022)$ \\
$\quad$ section & & & & & \\
Cortex area $\left(\mathrm{mm}^{2}\right)$ & $19.71 \pm 2.36$ & $16.35 \pm 1.77(-17 \%, p=0.0104)$ & & $52.52 \pm 4.40$ & $47.65 \pm 7.78(-9 \%, \mathrm{~ns})$ \\
Number of glomeruli/mm & & $9.08 \pm 1.24$ & $9.01 \pm 1.10(-1 \%, \mathrm{~ns})$. & & $3.92 \pm 0.31$ & $3.45 \pm 0.35(-12 \%, p=0.022)$ \\
\hline
\end{tabular}

Data are presented as mean $\pm \mathrm{SD}(p$ Dex $v s$ Sal $)$.

ns, not significant.

used. The pups were born on d 21-22 of gestation. On the day of birth, male pups from the same litters were randomly assigned to the treatment group and control group.

Experimental design. Rat pups were injected i.p. with Dex (Dexamethasone Sodium Phosphate; BUFA, Uitgeest, The Netherlands) on d $1(0.5 \mathrm{mg} / \mathrm{kg}$ body weight), d $2(0.3 \mathrm{mg} / \mathrm{kg}$ body weight $)$, and $\mathrm{d} 3(0.1 \mathrm{mg} / \mathrm{kg}$ body weight $)$ after birth. This treatment was based on a 21-d tapering treatment given to preterm infants to prevent or reduce CLD (19). Rat pups treated with equal volumes (10 $\mu \mathrm{L} / \mathrm{g}$ body weight) of sterile pyrogen-free saline (Sal) at d 1,2, and 3 served as control group. The rats (total $n=75$ ) were killed at $\mathrm{d} 2[n=$ (Sal versus Dex) 8 versus 8], $4(n=9$ versus 8$)$, and $7(n=7$ versus 6$)$ after birth, and at 4 ( $n=8$ versus 8$)$ and $50(n=7$ versus 6$)$ weeks of age. Rats were killed by decapitation. Body weight was measured before sacrifice. The kidneys were harvested and weighted and immediately immersion fixed in buffered formalin $4 \%$. The kidneys were further dissected after $24 \mathrm{~h}$ of fixation.

Histopathological assessment of renal damage. After formalin fixation, the kidneys were cut transversally in two equal parts and embedded in paraffin. The paraffin-embedded kidneys were then cut into $2 \mu \mathrm{m}$ sections for Periodic Acid Schiff (PAS) and immunohistochemical staining.

Kidney sections of 50-wk-old rats were used for renal damage measurements using ED-1 staining (macrophages) and desmin staining (activated podocytes).

After deparaffinization and blocking of endogen peroxidase activity, sections were heated in citrate buffer in an autoclave sterilizer for antigen retrieval $(10 \mathrm{mMol}$ and $\mathrm{pH}$ 6.0). Incubation with ED-1 antibody for $1 \mathrm{~h}$ (monoclonal $\alpha$-mouse antibody, kindly provided by Ed Döpp, Department of Cell Biology, Free University, Amsterdam, the Netherlands, diluted 1:2000) was followed by incubation with Rabbit Anti Mouse Peroxidase (polyclonal RAMPO, DAKO, Denmark, 1:100) in 10\% normal rat serum (IMGEN USA) for $30 \mathrm{~min}$, and then with Swine Anti Rabbit Peroxidase (polyclonal SWARPO, DAKO, Denmark, 1:100) in 10\% normal rat serum (IMGEN, USA) for $30 \mathrm{~min}$. Nova Red (Vector NovaRED, Vector Laboratories, CA) was used for color development and Mayer's Hematoxilin solution (Merck, Germany) was used for counter staining of nuclei.

After deparaffinization and blocking of endogen peroxidase activity, sections were heated in citrate buffer $(10 \mathrm{mMol}$ and $\mathrm{pH} 6.0)$ and boiled for $20 \mathrm{~min}$ for antigen retrieval. Anti-desmin antibody (monoclonal $\alpha$-mouse antibody, Biogenex, San Raman, CA; 1:100) was applied for $1 \mathrm{~h}$ and was followed by incubation with RAMPO 100) in 10\% normal rat serum (IMGEN USA) for 30 min, and then with polymeric horseradish peroxidase-linker antibody (PowerVision, Immunologic, Klinipath, The Netherlands, ready to use) for $30 \mathrm{~min}$ Nova Red (Vector NovaRED, Vector Laboratories, CA) was used for color development and Mayer's Heamatoxilin solution was used for counterstaining (Merck, Germany).

Measurements. All the measurements mentioned later were performed in a blinded fashion as to treatment group.

Proliferative activity was measured in kidney sections of rats of 2, 4, and $7 \mathrm{~d}$ of age by the method described by De Vries et al.(14). Briefly, the number of mitotic spindles per $\mathrm{mm}^{2}$ ("mitotic index") was counted in 15 consecutive high-power fields of the subcapsular area (nephrogenic zone) of the kidney at $400 \times$ magnification (total area $=0.23 \mathrm{~mm}^{2}$ ).

Glomerular density was determined using a modification of the method described by Tsuboi et al.(20) to approach nephron number. Glomerular density (number of glomeruli $/ \mathrm{mm}^{2}$ cortex area) was determined in PAS stained kidney sections of 4 - and 50-wk-old rats and determined by counting the number of glomeruli in one central transverse cross-section of the kidney at low-power magnification $(20 \times)$. The number of glomeruli was divided by the total cortex area of the same section as measured using image analysis software (ImageJ, Cell Imaging Facility Toronto, Toronto).

Renal damage in 50-wk-old rats was assessed by ED-1 staining to determine the number of inflammatory foci and by Desmin staining to determine podocyte stress.

An inflammatory focus was defined as an aggregate of inflammatory cells containing at least 10 lymphocytes and at least one ED-1 positive cell. Inflammatory foci were counted in 20 high-power fields $(200 \times$ magnification, total area of $1.84 \mathrm{~mm}^{2}$ ) of the cortex in a single cross-section of the kidney and expressed as number of inflammatory foci per $\mathrm{mm}^{2}$ cortex.

The number of ED-1 positive macrophages is associated with the severity of kidney damage (21). In brief, ED-1 positive cells were counted in 20 high power fields $\left(200 \times\right.$, total area of $\left.1.84 \mathrm{~mm}^{2}\right)$ of renal cortex in one crosssection and expressed as number of ED-1 positive cells per $\mathrm{mm}^{2}$ cortex area.

In the rat, renal damage can also manifest as desmin expression in stressed podocytes (21). In brief, 50 glomeruli per kidney were analyzed at a magnification of $400 \times$. Per glomerulus, a desmin score of 0 reflects staining of $0-5 \%$ of the glomerular cross sectional surface area; one reflects $5-25 \%$ staining; two reflects $25-50 \%$ staining; three reflects $50-75 \%$ staining; and four reflects $>75 \%$ staining. The podocyte stress score was defined as the mean desmin score of 50 glomeruli.

Statistical analysis. All data are expressed as mean \pm SD. Differences between the groups are determined with the Mann-Whitney $U$ test using SPSS versus 15.0.1. A $p<0.05$ was considered statistically significant.

\section{RESULTS}

Anatomical parameters. At d 2, bodyweight was lower in the Dex-treated rat pups but kidney weight was not different between the Dex and Sal group. At d 4, the body weight and kidney weight were 34 and $29 \%$ lower in Dex compared with Sal-treated rat pups. At d 7, this difference was 22 and $11 \%$, respectively. Table 1 summarizes these results.

At 4 wk of age, body weight and kidney weight was 16 and $24 \%$ lower in Dex compared with Sal rats $(p<0.001$ and $p<$ 0.001 , respectively). At $50 \mathrm{wk}$ of age, the body and kidney weight in the two groups were not different anymore (Table 2). 
Proliferative activity. Proliferative activity was determined by counting the number of mitotic spindles per $\mathrm{mm}^{2}$ subcapsular cortical area. Figure 1 shows representative microscopic pictures of the subcortical area of kidneys from 2-, 4-, and 7-d-old rats. There were less mitotic figures in the Dex-treated compared with Sal-treated pups: $9.1 \pm 2.4$ versus $16.4 \pm 4.0$ $(p<0.001)$ on $\mathrm{d} 2$ and $14.8 \pm 4.3$ versus $20.9 \pm 8.5(p<$ $0.05)$ on d 4 (mean \pm SD. Dex versus Sal). On d 7, a similar trend was observed, but the difference was no longer significant $(p=0.7)$ (Fig. 2).

Kidney size, glomerular density, and estimated nephron number in 4-wk-old and 50-wk-old rats. In 4-wk-old rats, there were less glomeruli per transversal kidney section in the Dex compared with the Sal group (18\% decrease, $p<0.001)$. The cortical surface area was smaller in 4-wk-old Dex rats (18\% decrease, $p<0.01$ ), but the number of glomeruli per $\mathrm{mm}^{2}$ cut surface cortex area was not significantly different. Fifty-week-old Dex rats, had 20\% less glomeruli per transversal kidney section $(p<0.05)$ than Sal rats, but differences in cortical surface area $(-9 \%)$ or kidney weight $(-4 \%)$ were not significant. Glomerular number per cortical surface area was $12 \%$ lower in Dex rats compared with Sal rats $(p<0.05)$. Table 2 summarizes these results.

Renal damage in 4-wk-old and 50-wk-old rats. Renal damage parameters were evaluated in 50-wk-old rats (illustrated in Figs. 3 and 4). At this age, the Dex-treated rats showed evidence of extensive kidney damage with foci of inflammatory cells, associated with sclerotic glomeruli, together with dilated tubuli with protein casts. In Sal rats, such changes were seen only sporadically. Dex rats had about 3.5 times more focal inflammatory infiltrates $(p<0.005), 3.3$ times more ED-1 positive macrophages $(p<0.05)$, and a 4.3 -fold higher glomerular podocyte stress score $(p<0.05)$.
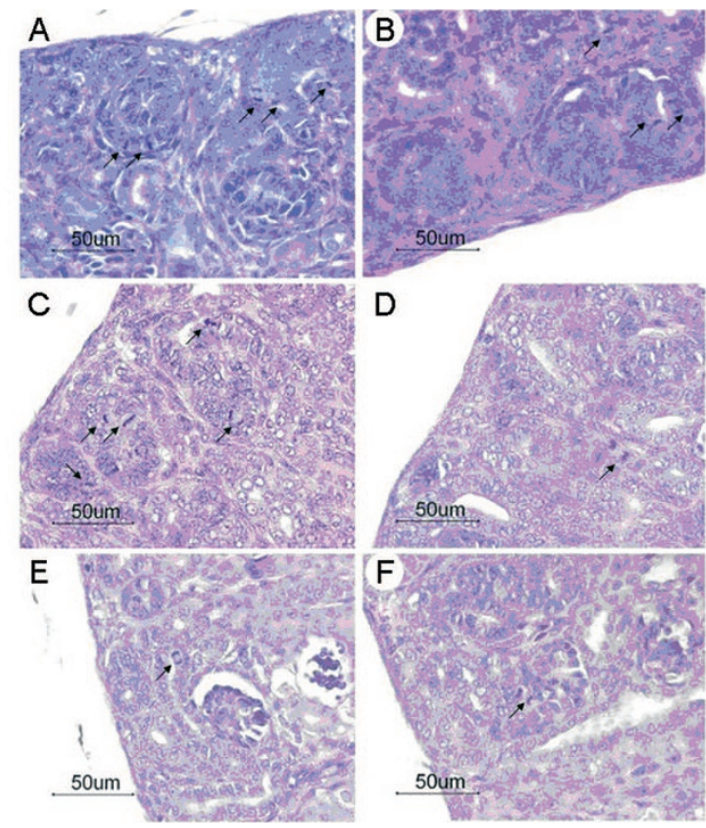

Figure 1. Histology of 2-, 4-, and 7-d-old rats neonatally treated with Sal and Dex. PAS staining of the subcapsular nephrogenic zone. A, 2-d-old Sal (control) rats; $B$, 2-d-old Dex rats; $C$, 4-d-old Sal rats; $D$, 4-d-old Dex rats; $E$, 7-d-old Sal rats; and $F, 7$-d-old Dex rats. Arrows indicate mitotic figures.

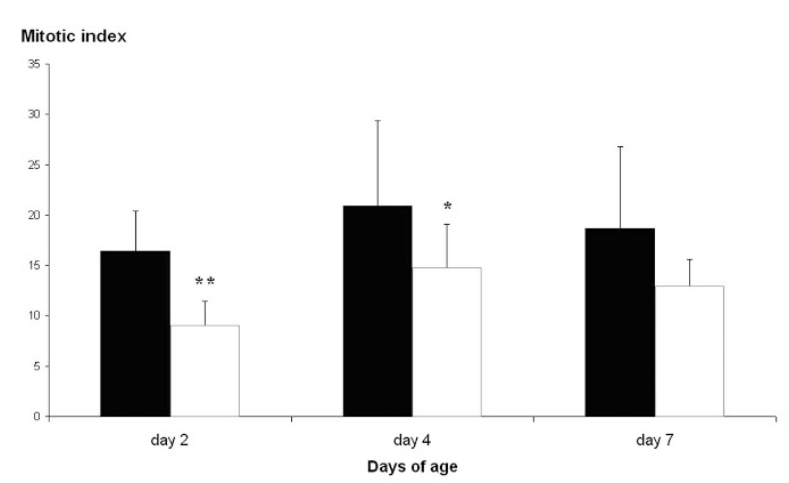

Figure 2. Proliferation of renal cells (mitotic index) of Sal- and Dex-treated neonatal rats. The mitotic index shows a significant decrease in proliferative activity of renal cells on $\mathrm{d} 2$ and 4 in Dex-treated rats (white bars) compared with the Sal (control) group (black bars). On d 7, no difference in mitotic index is found between the two groups. Data are presented as mean $\pm \mathrm{SD}$ $* * p<0.001 ; * p<0.05$ Dex $v s$ Sal. Mitotic index $=$ number of mitotic spindles in a subcordial area per $\mathrm{mm}^{2}$ (see Materials and Methods).
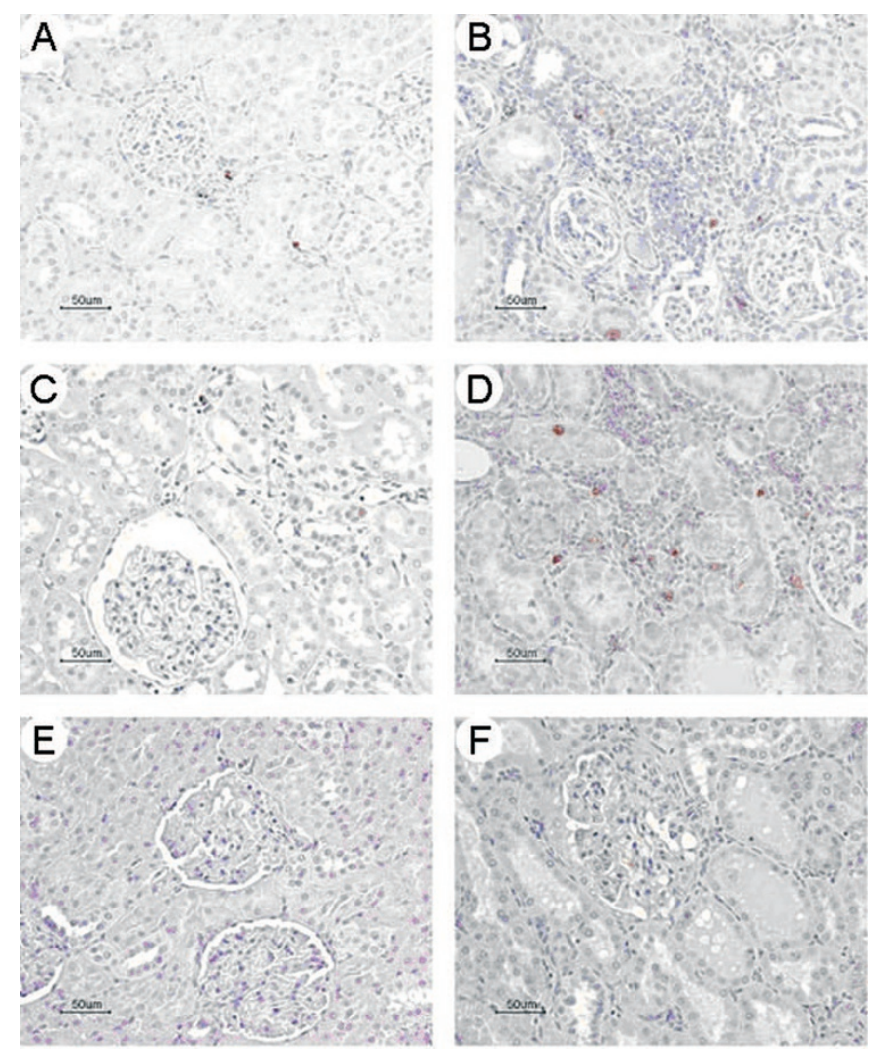

Figure 3. Histology of 50-wk-old rats neonatally treated with Sal or Dex. First row, in the ED-1 staining, the number of inflammatory foci was determined. In Sal (control) $(A)$ one small inflammatory foci is seen. In Dex-treated rats $(B)$, more and much larger foci are seen with more ED-1 positive cells. Second row, dex-treated rats $(D)$ show much more ED-1 positive cells compared with the Sal-treated rats $(C)$. Third row, in Sal- treated rats $(E)$, no desmin positive podocytes are seen (score 0 ). In Dex-treated rats $(F)$, some desmin positive podocytes can be found (score 3 ).

\section{DISCUSSION}

In this study, we aimed to investigate the short-term and long-term effects on the kidney of neonatal Dex treatment in rats, as a model for neonatal Dex treatment in preterm infants for prevention or reduction of CLD. The major findings were 

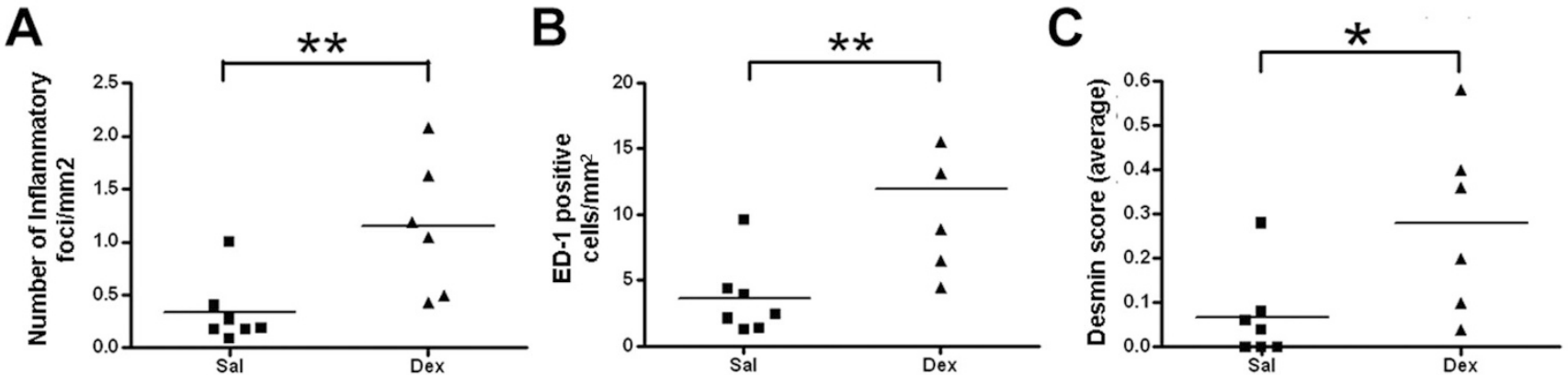

Figure 4. Renal damage scores of 50-wk-old rats treated with Sal or Dex. Assessment of renal damage in 50-wk-old rats shows a significant difference seen between Sal- and Dex-treated rats in the number of $(A)$ inflammatory foci $/ \mathrm{mm}^{2}(p=0.0047),(B)$ ED-1 positive cells $/ \mathrm{mm}^{2}(p=0.0082)$, and in $(C)$ desmin score $(p=0.0221)$. Data are presented as mean $(p \operatorname{Dex} v s \mathrm{Sal})$.

that neonatal Dex treatment on $\mathrm{d} 1,2$, and 3 in rats causes a temporary suppression of proliferative activity of renal cells, which was associated with lower kidney weight and nephron number and extensive renal damage in adult life and during aging. These findings are in agreement with previous observations in the heart. Also in the myocardium, a suppression of proliferative activity was found on $\mathrm{d} 2$ and 4 , and there was no catch-up growth at later days (14). In addition, total body weight was lower in Dex-treated rats, which suggests that neonatal Dex treatment had a negative effect on organ growth and development. This was similar to findings in earlier studies (13-15). The differences in weight may be partially due to nutritional problems in the days after the neonatal treatment. Although we suggest that the effects on the kidney were induced by a direct effect of Dex treatment, this study cannot definitely exclude the possibility that the Dex-induced growth restriction in itself, during this very active phase of nephrogenesis, may have indirectly influenced kidney weight and nephron formation $(22,23)$.

In the human kidney, glomerulogenesis is completed around wk 34-36 of gestation, well before a term delivery (wk $37-40$ ), and postnatal development is limited to maturation and growth (24). In rats, however, glomerulogenesis continues beyond the first week after birth. Therefore, newborn rats may constitute a model for early postnatal life of preterm infants, which implies that our findings are indicative of potential negative side effects of current Dex treatment. The mechanism behind suppression of renal cell proliferation by Dex is incompletely understood but probably involves repression of NF-kB and AP-1 (25). At 4 wk of age, Dex-treated rats still had lower body weight than controls, and the number of glomeruli and kidney weight were reduced proportionally. Thus, there was no compensatory nephron hypertrophy. However, in older rats (50 wk), bodyweight of Dex rats and controls was similar, as was kidney weight, whereas Dex rats had a lower cortical glomerular density, consistent with nephron hypertrophy, and showed a trend toward lower total cortical surface area. At this age, also renal damage parameters were significantly higher in Dex rats, consistent with observations in a previous report by Liu et al.(18).

There are several possible causes of renal damage. The reduced number of nephrons might have suffered from hyperfiltration and increased sodium reabsorption. Also, previous studies have demonstrated that antenatal glucocorticoid exposure induces dilatation of both afferent and efferent arterioles, with concomitant increase of GFR and proximal sodium reabsorption, in association with increased activity and expression of $\mathrm{Na}+\mathrm{K}+$ ATPase $(24,26)$. Stubbe et al.(27) reported that also postnatal Dex administration can lead to increased sodium reabsorption, and a permanent increase of $\mathrm{Na}+\mathrm{K}+$ $2 \mathrm{Cl}$ cotransporter, and of $\mathrm{Na}+\mathrm{K}+\mathrm{ATPase} \alpha 1$. Heart failure has a major negative impact on the kidney, and renal failure is a major progression factor of cardiovascular disease. This mechanism of mutual aggravation of pathologies might be involved also in the severity of organ damage observed in adult rats in this and previous studies of perinatal Dex treatment. However, the early preclinical changes that we have observed in both the heart and the kidney indicate a primary treatment-related pathologic effect of neonatal Dex treatment in both organs.

In conclusion, Dex treatment of neonatal rats leads to lower nephron numbers and extensive renal damage in aging rats. This is a warning that current practice of Dex treatment in prematurely born infants might increase the risk of developing renal damage in later life, as has been suggested previously for the impact of this therapy on the developing heart.

Acknowledgments. We thank Ms. Dionne vd Giessen for expert technical assistance.

\section{REFERENCES}

1. Ehrenkranz RA, Walsh MC, Vohr BR, Jobe AH, Wright LL, Fanaroff AA, Wrage LA, Poole K 2005 Validation of the National Institutes of Health consensus definition of bronchopulmonary dysplasia. Pediatrics 116:1353-1360

2. Coalson JJ 2003 Pathology of new bronchopulmonary dysplasia. Semin Neonatol 8:73-81

3. Committee on Fetus and Newborn 2002 Postnatal corticosteroids to treat or prevent chronic lung disease in preterm infants. Pediatrics 109:330-338

4. Kinsella JP, Greenough A, Abman SH 2006 Bronchopulmonary dysplasia. Lancet 367:1421-1431

5. Barrington KJ 2001 Postnatal steroids and neurodevelopmental outcomes: a problem in the making. Pediatrics 107:1425-1426

6. Stark AR, Carlo WA, Tyson JE, Papile LA, Wright LL, Shankaran S, Donovan EF, Oh W, Bauer CR, Saha S, Poole WK, Stoll BJ 2001 Adverse effects of early dexamethasone in extremely-low-birth-weight infants. National Institute of Child Health and Human Development Neonatal Research Network. N Engl J Med 344:95-101

7. Yeh TF, Lin YJ, Lin HC, Huang CC, Hsieh WS, Lin CH, Tsai CH 2004 Outcomes at school age after postnatal dexamethasone therapy for lung disease of prematurity. N Engl J Med 350:1304-1313

8. Jobe AH 2004 Postnatal corticosteroids for preterm infants-do what we say, not what we do. N Engl J Med 350:1349-1351 
9. Kamphuis PJ, Croiset G, Bakker JM, van Bel F, Van Ree JM, Wiegant VM 2004 Neonatal dexamethasone treatment affects social behaviour of rats in later life. Neuropharmacology 47:461-474

10. Raff H 2004 Neonatal dexamethasone therapy: short-and long-term consequences Trends Endocrinol Metab 15:351-352

11. Shinwell ES, Eventov-Friedman S 2009 Impact of perinatal corticosteroids on neuromotor development and outcome: review of the literature and new metaanalysis. Semin Fetal Neonatal Med 14:164-170

12. Kamphuis PJ, de Vries WB, Bakker JM, Kavelaars A, van Dijk JE, Schipper ME, van Oosterhout MF, Croiset G, Heijnen CJ, van Bel F, Wiegant VM 2007 Reduced life expectancy in rats after neonatal dexamethasone treatment. Pediatr Res 61:72-76

13. de Vries WB, van der Leij FR, Bakker JM, Kamphuis PJ, van Oosterhout MF, Schipper ME, Smid GB, Bartelds B, van Bel F 2002 Alterations in adult rat heart after neonatal dexamethasone therapy. Pediatr Res 52:900-906

14. de Vries WB, Bal MP, Homoet-van der Kraak P, Kamphuis PJ, van der Leij FR, Baan J, Steendijk P, de Weger RA, van Bel F, van Oosterhout MF 2006 Suppression of physiological cardiomyocyte proliferation in the rat pup after neonatal glucocorticosteroid treatment. Basic Res Cardiol 101:36-42

15. Bal MP, de Vries WB, Steendijk P, Homoet-van der Kraak P, van der Leij FR, Baan J, van Oosterhout MF, van Bel F 2009 Histopathological changes of the heart after neonatal dexamethasone treatment: studies in 4-, 8- and 50-week-old rats. Pediatr Res 66:74-79

16. Martins JP, Monteiro JC, Paixao AD 2003 Renal function in adult rats subjected to prenatal dexamethasone. Clin Exp Pharmacol Physiol 30:32-37

17. Ortiz LA, Quan A, Weinberg A, Baum M 2001 Effect of prenatal dexamethasone on rat renal development. Kidney Int 59:1663-1669

18. Liu Y, van Goor H, Havinga R, Baller JF, Bloks VW, van der Leij FR, Sauer PJ, Kuipers F, Navis G, de Borst MH 2008 Neonatal dexamethasone administration causes progressive renal damage due to induction of an early inflammatory response. Am J Physiol Renal Physiol 294:F768-F776

19. Kamphuis PJ, Bakker JM, Broekhoven MH, Kunne C, Croiset G, Lentjes EG, Tilders FJ, van Bel F, Wiegant VM 2002 Enhanced glucocorticoid feedback inhibition of hypothalamo-pituitary-adrenal responses to stress in adult rats neonatally treated with dexamethasone. Neuroendocrinology 76:158-169

20. Tsuboi N, Kawamura T, Ishii T, Utsunomiya Y, Hosoya T 2009 Changes in the glomerular density and size in serial renal biopsies during the progression of $\operatorname{IgA}$ nephropathy. Nephrol Dial Transplant 24:892-899

21. Attia DM, Ni ZN, Boer P, Attia MA, Goldschmeding R, Koomans HA, Vaziri ND, Joles JA 2002 Proteinuria is preceded by decreased nitric oxide synthesis and prevented by a NO donor in cholesterol-fed rats. Kidney Int 61:1776-1787

22. Luyckx VA, Brenner BM 2005 Low birth weight, nephron number, and kidney disease. Kidney Int Suppl S68-S77

23. Moritz KM, Singh RR, Probyn ME, Denton KM 2009 Developmental programming of a reduced nephron endowment: more than just a baby's birth weight. Am J Physiol Renal Physiol 296:F1-F9

24. Jahnukainen T, Chen M, Berg U, Celsi G 2001 Antenatal glucocorticoids and renal function after birth. Semin Neonatol 6:351-355

25. Bladh LG, Liden J, Pazirandeh A, Rafter I, Dahlman-Wright K, Nilsson S, Okret S 2005 Identification of target genes involved in the antiproliferative effect of glucocorticoids reveals a role for nuclear factor-(kappa)B repression. Mol Endocrinol 19:632-643

26. Cattarelli D, Chirico G, Simeoni U 2002 Renal effects of antenatally or postnatally administered steroids. Pediatr Med Chir 24:157-162

27. Stubbe J, Madsen K, Nielsen FT, Skott O, Jensen BL 2006 Glucocorticoid impairs growth of kidney outer medulla and accelerates loop of Henle differentiation an urinary concentrating capacity in rat kidney development. Am J Physiol Renal Physiol 291:F812-F822 